\title{
Exploring Student Difficulties with Buoyancy
}

\author{
DJ Wagner*, Elizabeth Carbone* and Ashley Lindow* \\ *Department of Physics, Grove City College, 100 Campus Drive, Grove City, PA 16127
}

\begin{abstract}
Our research group is developing a standardized fluids assessment, covering buoyancy and pressure. Understanding buoyancy requires a battery of skills and knowledge, and we have designed questions to probe understanding of background concepts such as density, incompressibility, and volume of fluid displaced. In this paper we will describe some of the buoyancy-related assessment questions, the misconceptions they probe, and the preliminary results from the beta version of the assessment.
\end{abstract}

Keywords: Buoyancy, Archimedes’ Principle, Fluids, Education Research.

PACS: 01.40.Fk, 01.40.G-

\section{INTRODUCTION}

As part of the development of a fluids statics diagnostic, we are examining student conceptions of buoyancy. We interviewed eight students at the end of the fall, 2010 semester, and we have analyzed longanswer explanations given by students taking drafts of our assessment. Distractors for our questions are based on conceptions identified by these methods, and by other studies [1-16]. Since few of those studies [1-5] involve students above the age of $15,{ }^{1}$ we wondered which difficulties identified in those studies would persist into college. Our assessment can then focus on these conceptions held by our target audience.

In this paper we discuss selected common student ideas about buoyancy and summarize their prevalence in our courses, as measured by various drafts of our diagnostic questions. Due to space constraints, we will focus on our diagnostic questions that explicitly address buoyant force and volume displaced, probing student difficulties concerning Archimedes’ Principle.

\section{PRIOR WORK}

Correctly answering a question involving Archimedes' Principle requires the activation of many resources [2]; thus a particular answer to a given diagnostic question could be chosen based on one of many thought processes or conceptions. Below we describe conceptions that suggest an incomplete understanding of Archimedes Principle, grouped by the (incorrect) physical outcome predicted by each conception. Conceptions are assigned labels, in (boldface) when defined and later referenced in italics.

i. Buoyant force (on fully-submerged object) is greater for objects of greater mass but same volume.

\footnotetext{
${ }^{1}$ Reference 5 includes in-service teachers, focuses on children.
}

This conclusion may be made directly ( $\mathbf{F}_{\mathbf{b}}$ mass) $[7,18]$, or by equating buoyant force and weight regardless of whether objects sink or float (weight) $[2,8]$. Other students may base this conclusion explicitly on a mass dependence of the volume of fluid displaced (VFD) on the object's mass ( $\mathbf{V}$ mass) [3,6,912,17] or density (density) [11-12,17].

ii. Buoyant force (on fully-submerged object) increases with depth in the fluid. Some students may arrive at this conclusion by misinterpreting VFD as the volume of the column of fluid above a submerged object (column above) [2,6,13]. Others may conclude that buoyant force increases as the pressure increases with depth (pressure) [2,17].

iii. Buoyant force (on fully-submerged object) decreases with depth in the fluid. This outcome is consistent with the buoyant force taken to be zero for an object at rest on the bottom of a container [14,18].

A common error by students is associating equilibrium position with applied force (position) $[2,8]$. Thus a block at equilibrium placed deeper in the water must be experiencing a smaller buoyant force than an identical block placed less deeply. Students could also choose this outcome by failing to distinguish buoyancy from buoyant force, saying that a larger buoyant force results in an object being "more buoyant;" thus the buoyant force on a floating object would be greater than a submerged object of the same size (buoyancy) [2]. Or, students may say that buoyant force depends upon the amount of water beneath the object pushing upwards (beneath) $[7,14]$.

iv. Buoyant force depends upon amount of fluid in container. The total volume of fluid surrounding an object is often connected to buoyant force (amt fluid) $[4,9,12,15]$. Students with this belief might suggest that fluid in a wider container but the same depth can support more weight [4].

$v$. Buoyant force depends upon orientation or shape of constant-volume object. Students may 
demonstrate a lack of awareness of conservation of volume by saying that reshaping or reorienting a constant-volume object can affect VFD (shape) $[3,16]$. Or, students may argue that reshaping an object to make it "flatter" will increase buoyant force because of the greater horizontal surface area experiencing an upwards force from the fluid (flatter) [4,14].

vi. Buoyant force depends upon other forces acting on the object. Students often treat buoyant force as varying with other forces acting on the object, such as tension or normal forces $\left(\mathbf{F}_{\mathbf{b}} \sim \mathbf{o t h e r} \mathbf{F}\right)$ [2,15].

vii. Erroneous treatment of buoyant force as fixed. Many students equate VFD to an object's total volume, even when the object is only partially submerged ( $\mathbf{V}$ tot) [2], making the buoyant force on an object independent of its state of submersion. Others may decide the buoyant force is fixed for a particular object without explicitly considering VFD (fixed) [15]. Or, students might apply compensation reasoning (compensation) [2], assuming that a smaller object in a denser fluid experiences the same buoyant force as a larger object in a rarer fluid, regardless of actual ratios.

viii. Misconsideration of the weight of fluid displaced (WFD). Students may ignore the effect of fluid density on buoyant force, not distinguishing between VFD and WFD (V only) [4,7]. Conversely, students might claim VFD itself depends upon the fluid density. (fluid rho) [2,11]. Or, they could say that the buoyant force on a floating object in equilibrium depends upon the fluid's density, rather than always equaling the object's weight (equilibrium) [15].

Another difficulty with WFD is equating VFD to the volume of the object that is not submerged ( $\mathbf{V}$ inverse) [15]. One of our interviewees expressed the belief that floating objects do not displace any fluid at all ( $\mathbf{V}$ zero). An approach we find particularly interesting is saying that the addition of an absorbent material to a fluid will lower the fluid level, essentially allowing for a negative VFD (negative) [5,19].

\section{OUR QUESTIONS}

The development of our assessment is an ongoing project, and we continually balance inclusion of conceptions with the desire to keep the assessment length reasonable, and to keep questions from becoming too complex. For the sake of completeness, we summarized all Archimedes-specific conceptions that we have identified in the prior section, but not all of those conceptions are addressed by the questions on our current draft of the diagnostic.

Figure 1 contains the full text of four questions from drafts of our diagnostic, questions which explicitly address the buoyant force from the perspective of Archimedes' Principle. (The "Strings" question was given in a different year than the other questions.) Table 1 lists answer options consistent with the previously-defined physical outcomes i through viii. Some options are specific enough to target individual conceptions within an outcome; for these items, the conception is in italics in the first column.

Looking at combinations of answers for the multiple-select "Tether" question might allow us to discriminate between the amt fluid conception and the depth-dependent conceptions, as shown in Table 1. Students who use both conceptions will unfortunately not be counted for the amt fluid conception but be included only in the "depth" category.

TABLE 1. Answer options for selected question associated with each physical result. If an "outcome" is in italics, the option targets that specific conception, rather than just identifying the outcome category.

\begin{tabular}{l|c|c|c|c}
\hline \multicolumn{1}{c|}{ Outcome } & Tether & Strgs & Bott & Disp \\
\hline i. $\mathrm{F}_{\mathrm{b}} \sim$ mass & $\mathrm{A}$ & & $\mathrm{A}$ & \\
\hline ii. $\mathrm{F}_{\mathrm{b}} \sim$ depth & $\mathrm{C}+\mathrm{F}(+\mathrm{H})^{2}$ & $\mathrm{C}$ & & $\mathrm{B}$ \\
\hline iii. $\mathrm{F}_{\mathrm{b}} \sim$ depth $^{-1}$ & $\mathrm{D}+\mathrm{G}$ & & $\mathrm{C}$ & $\mathrm{A}$ \\
\hline iv. amt fluid & $\mathrm{C}$ or D & & & \\
\hline v. flatter & $\mathrm{H}$ & & & \\
\hline vi. $F_{b} \sim$ other $F$ & $\mathrm{I}$ & A or B & & \\
\hline vii. $V$ tot & & & & $\mathrm{E}$ \\
\hline viii. $V$ inverse & & & & $\mathrm{D}$ \\
\hline
\end{tabular}

\section{DATA}

Various drafts of our diagnostic questions have been given to three introductory courses at Grove City College (GCC): a calculus-based course ("calc") for engineers and physics majors, a trig-based course ("trig") for biology and chemistry majors, and a concept-based course ("cnpt”) for non-science majors.

Results for the four questions of interest to this paper are displayed in Fig. 2, grouped by outcome. For ease of reading, we have included only post-instruction data; examination of the pre-instruction data does not lead us to draw additional conclusions for these questions at this stage of the assessment development.

Students can hold multiple (sometimes conflicting) conceptions, so we have counted any response to the multiple-select Tether question that included the listed option or combination. When separating the amt fluid conception from the depth-dependent conceptions, we count students who chose $\mathrm{C}$ and not F, or D and not G. The prevalence of the flatter conception was distinguished from the depth-dependent conceptions by counting students who chose $\mathrm{H}$ but not $\mathrm{F}$.

Because very few students chose the answers consistent with outcomes iii, iv, and $\mathrm{v}$ (with one

\footnotetext{
${ }^{2}$ The " $+\mathrm{H}$ " is in parentheses, since students may not all assume that rotating the block moves it deeper. Either of the answer combinations CF or CFH is attributed to $\mathrm{F}_{\mathrm{b}} \sim$ depth.
} 
"Tether" (F12/S13 only): A rectangular block is tethered to the bottom of a container of water, as shown to the right. Which of the following would increase the buoyant force on the block? Choose all which apply.

A. Using a block of a larger mass but same size. $\mathbf{F}_{\mathbf{b}} \sim \operatorname{mass}$

B. Using a block of a larger size but same mass.

C. Adding more water to the tank. $\mathbf{F}_{\mathbf{b}} \sim$ depth (if $\mathbf{F}$ chosen also); amt fluid (if alone)

D. Removing some water from the tank (while keeping the block completely submerged. $\mathbf{F}_{\mathbf{b}} \sim \mathbf{d e p t h}^{-1}$ (if $\mathbf{G}$ chosen also); amt fluid (if alone)

E. Replacing the water in the tank with liquid mercury (which has a much higher density than water and would weigh much more).

F. Shortening the tether so the block was closer to the bottom of the container. $\mathbf{F}_{\mathbf{b}} \sim$ depth (if $\mathbf{C}$ chosen also)

G. Lengthening the tether so the block was closer to the surface (but still completely submerged). $\mathbf{F}_{\mathbf{b}} \sim \mathbf{d e p t h}^{-1}$ (if $\mathbf{D}$ chosen also)

H. Rotating the block so the tether attached to the longer side. $\mathbf{F}_{\mathbf{b}} \sim \mathbf{d e p t h}$ (if $\mathbf{C}$ and $\mathbf{F}$ chosen also)

I. None, since the buoyant force is zero, due to the tether holding the block in place. $\mathbf{F}_{\mathbf{b}} \sim \mathbf{o t h e r} \mathbf{F}$

"Strings" (F10-S11 only): Three identical blocks are at rest in a water-filled aquarium as shown to the right. Blocks B and C are attached to taut strings. Compare the buoyant force (the net force the water exerts) on the three blocks.

A. The buoyant force is greatest on $A$ and smallest on C. $\mathbf{F}_{\mathbf{b}} \sim$ other $\mathbf{F}$

B. The buoyant force is greatest on $\mathrm{C}$ and smallest on $\mathrm{A} . \mathbf{F}_{\mathbf{b}} \sim$ other $\mathbf{F}$

C. The buoyant force is equally great on $\mathrm{A}$ and $\mathrm{B}$ and smallest on C. $\mathbf{F}_{\mathbf{b}} \sim \mathbf{d e p t h}$

D. The buoyant force is the same for all three blocks.

E. It cannot be determined from the information given

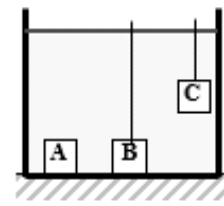

"Bottom": Three blocks of different masses but the same shape and size lie on the bottom of an oil-filled aquarium as shown to the right. Compare the buoyant force (the net force the oil exerts) on the three blocks, if block A has the greatest mass and block $\mathrm{C}$ the least mass.

A. The buoyant force is greatest on $\mathrm{A}$ and smallest on C. $\mathbf{F}_{\mathbf{b}} \sim$ mass

B. The buoyant force is greatest on $\mathrm{C}$ and smallest on $\mathrm{A}$.

C. The buoyant force is zero on all three blocks, since they are on the bottom. $\mathbf{F}_{\mathbf{b}} \sim \mathbf{d e p t h}^{\mathbf{1}}$

D. The buoyant force is the same for all three blocks but not zero.

E. It cannot be determined from the information given.

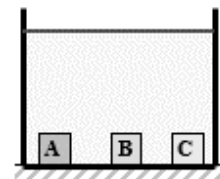

"Displaced": Three blocks of different masses but the same shape and size are at rest in water as shown to the right. How does the volume of water displaced by the blocks compare?

A. Block A displaces the greatest volume water and block $\mathrm{C}$ displaces the least. $\mathbf{F}_{\mathbf{b}} \sim \mathbf{d e p t h}^{-1}$

B. Block $\mathrm{C}$ displaces the greatest volume water and block A displaces the least. $\mathbf{F}_{\mathbf{b}} \sim$ depth

C. The volume of water displaced is equally large for B and C and smallest for A.

D. The volume of water displaced is greatest for $\mathrm{A}$ and equally small for $\mathrm{B}$ and $\mathrm{C}$. $\mathrm{V}$ inverse

E. The volume of water displaced is the same for all three blocks. $V$ tot

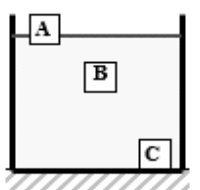

FIGURE 1. Assessment questions explicitly addressing the buoyant force from an Archimedes approach. Conceptions probed by each option have been added in bold-face after the response.
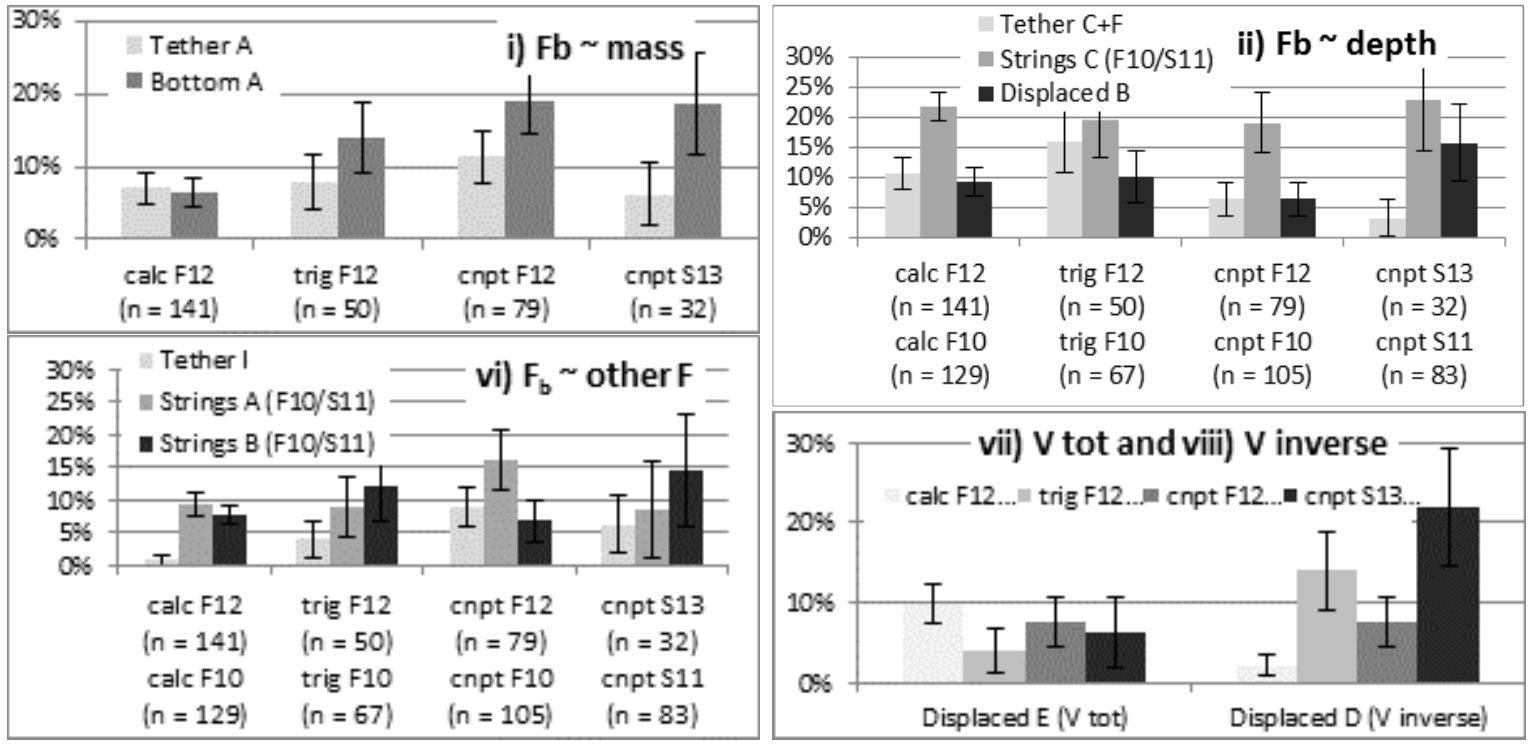

FIGURE 2. \% students choosing options associated with outcome/results from Table 1. Outcomes iii, iv, and v are not presented due to lower numbers. Some data labels indicate two $n$, because the "Strings" question was given in a prior year. Error bars represent standard error assuming binomial ( 1 if choosing option, 0 if not) approach. 
exception), those results are not graphed. The exception involves outcome iii $\left(\mathrm{F}_{\mathrm{b}} \sim \mathrm{depth}^{-1}\right)$ : Option $\mathrm{C}$ of the Bottom question was chosen by $9-18 \%$ of students, depending upon the class. The other two options consistent with outcome iii were only chosen by $0-8 \%$ of students. No more than $13 \%$ of students in any course chose Tether $\mathrm{C}$ without $\mathrm{F}$, and fewer than $5 \%$ of students in any class chose Tether D without $G$ (amt fluid). No student chose $\mathrm{H}$ without F (flatter).

\section{CONCLUSIONS}

Several previously-identified conceptions appear not to be prevalent in our students. Fewer than 10\% of students in each of our courses selected answers consistent with $V$ tot, and none chose the flatter option. amt fluid was chosen by no more than $13 \%$ of students in a course. While this result could be depressed due to the way we are extracting it, the results of additional questions on the assessment [20] are consistent with this conclusion. Further study with more samples is necessary, but this data suggests that our assessment need not focus on these three conceptions.

Only one [2] of the studies [2,7,8] identifying conceptions associated with $\mathrm{F}_{\mathrm{b}} \sim$ depth $^{-1}$ involved college students, so perhaps students "grow out" of this conclusion. Further study of why students choose Bottom $C$ but not Displaced $A$ or Tether $D+G$ is needed.

Comparing the "Strings" options A/B results to the "Tether" option I results (outcome vi) suggests that students might view the buoyant force as dependent upon, but not eliminated by, tension. This outcome is only marginally more popular than amt fluid; inclusion in the assessment will depend upon future study. The other three outcomes $\left(\mathrm{F}_{\mathrm{b}} \sim\right.$ mass, $\mathrm{F}_{\mathrm{b}} \sim$ depth, and $\mathrm{V}$ inverse) seemed more popular; we will try to distinguish between different conceptions associated with these outcomes in the final assessment.

Our assessment should include redundancy. But the results for outcome iii and outcome i ( $\mathrm{F}_{\mathrm{b}} \sim$ mass) demonstrate that what we might think are redundant options (such as Tether A and Bottom A, or Bottom C and Displaced A) do not necessarily prove similar to the students. Additionally, students frequently hold simultaneous conflicting views. About $1 / 4$ of students in most classes (and almost $1 / 2$ of trig students) chose both at least one correct answer to Tether along with at least one incorrect answer.

This study illustrates the difficulty in probing specific conceptions rather than physical outcomes, but we believe the information will help us as we determine which conceptions to probe in the final version of the assessment. Future steps will include requesting explanations on Tether and Bottom to probe outcome i, de-emphasizing conceptions that are not chosen by at least 5\% of our students, and probing the "zero on the bottom” idea.

\section{ACKNOWLEDGMENTS}

The authors would like to thank the following members of the GCC PER group for their contributions: Zachary Bazan, Sam Cohen, Kathryn Merrymon, Adam Moyer, and Jason Wetstone. Partial support for this work has come from the GCC Swezey Scientific Instrumentation and Research Fund.

\section{REFERENCES}

1. U. Besson, Int J Sci Educ 26, 1683-1714 (2004).

2. M.E. Loverude, C.H. Kautz, and P.R.L. Heron, Am. J. Phys. 71, 1178-1187 (2003).

3. J. McKinnon, J. Geol. Educ. 19, 218 (1971).

4. E. R. Duckworth, "Inventing density." in Grand Forks, ND, North Dakota Study Group on Evaluation, Center for Teaching and Learning, University of North Dakota, 1986. (WWW document http://www.exploratorium.edu/ ifi/resources/classroom/inventingdensity.html Downloaded 6/12/2011)

5. L. E. Klopfer, A. B. Champagne, and S. D. Chaiklin, Sci. Ed. 76, 597-614 (1992).

6. D. Wong, C. C. Lim, S. Munirah and S. K. Foong, poster at 2010 Physics Education Research Conference. (WWW document http://www.compadre.org/per/perc/2010/ Detail.cfm?id=3543 Downloaded 7/5/2012.)

7. E. Mullet and A. Montcouquoil, Int J Sci Educ 10, 285301 (1988).

8. O. A. Dentici, M. G. Grossi, L. Borghi,, A. D. Ambrosis, \& C. I. Massara, Eur J Sci Educ 6(3), 235-243 (1984).

9. B. Inhelder and J. Piaget, The growth of logical thinking from childhood to adolescence. New York: Basic Books, 1958.

10. E. D. Gennaro, Sch Sci \& Math 81(5), 399-404 (1981).

11. E. D. Gennaro, Sch Sci \& Math 66(6), 559-560 (1966).

12. Y. Yin, M.K. Tomita, and R.J. Shavelson, Science Scope 34, 34-39 (2008).

13. C. Smith, J. Snir, \& L. Grosslight, Cognition \& Instruction, 9(3), 221-283 (1992).

14. S. Gang, Phys. Teach. 33(9), 346-354 (1995).

15. S. Ünal and B. Coştu, Problematic issue for students: Does it sink or float? Asia-Pacific Forum on Science Learning and Teaching 6(1) (2005).

16. F. Biddulph and R. Osborne, Res Sci Ed 14, 114-124 (1984).

17. These conceptions were expressed by our interview subjects in Fall, 2010.

18. The authors and their colleagues have heard many students express these conceptions during a lab on buoyancy.

19. These conceptions were contained within written explanations by students taking our assessment.

20. D. Wagner, et. al., poster presented at Physics Education Research Conference 2012, Philadelphia, PA, 2012, (unpublished). 